Eur Neurol 2001;45:57-58

\title{
Memantine Treatment in Alcohol Dementia: Rapid PET Changes and Clinical Course
}

\author{
U.W. Preussa , M. Bahlmann ${ }^{\text {a }}$ P. Bartenstein ${ }^{\text {b }, ~ C . G . ~ S c h u ̈ t z ~}{ }^{a}$, \\ M. Soyka \\ a Psychiatrische Klinik und Poliklinik, Ludwig-Maximilians- \\ Universität München und ${ }^{\mathrm{b}}$ Klinik und Poliklinik für \\ Strahlentherapie und Radiologische Onkologie, Technische \\ Universität München, Deutschland
}

Alcohol dementia (ALD) usually follows long-term alcohol dependence. The major psychiatric features of ALD are general loss of short- and long-term memory combined with disturbances in concentration and a decrease in intellectual capacity, all of which persist during abstinence. Despite its clinical importance, few studies have been published up to now on medical treatment of ALD and other alcohol-induced mental disorders [1]. The central nervous glutamatergic system, with its N-methyl- $D$-aspartate (NMDA) receptors, is suggested to be involved in toxic neuronal loss [2] due to an increased glutamatergic neurotransmission during repeated alcohol withdrawal and may subsequently contribute to the development of ALD. The low-affinity NMDA receptor antagonist memantine (adamantine, 1-amino-3,5-dimethyladamantine hydrochloride, CAS 41100-52-1) modulates the glutamatergic system most likely through $\mathrm{Ca}^{2+}$ influx blockage of NMDA receptors [3] and shows positive effects on memory in the treatment of other psychogeriatric disorders with memory loss such as Alzheimer's dementia (AD) or Parkinson's disease [4].

We would like to report the case of a 71-year-old female patient with chronic alcoholism for at least 30 years. She was found neglected in her home and was admitted to our ward from an emergency unit with symptoms of disorientation, apraxia, ataxia, severe short- and long-term memory disturbances, deficits in intellectual capacity and concentration. There were no signs of electrolyte imbalance on admission. A further thorough neurological investigation and NMR showed a general atrophy without severe basal brain damage of Wernicke encephalopathy. No other signs of Wernicke encephalopathy, i.e. ophthalmoplegia or evidence of hepatic encephalopathy such as flapping tremor, were found. Ultrasound of brain arteries showed some plaques without hemodynamic compromise. After excluding Alzheimer's disease or vascular dementia, the diagnosis of ALD according to ICD10 criteria was made. 


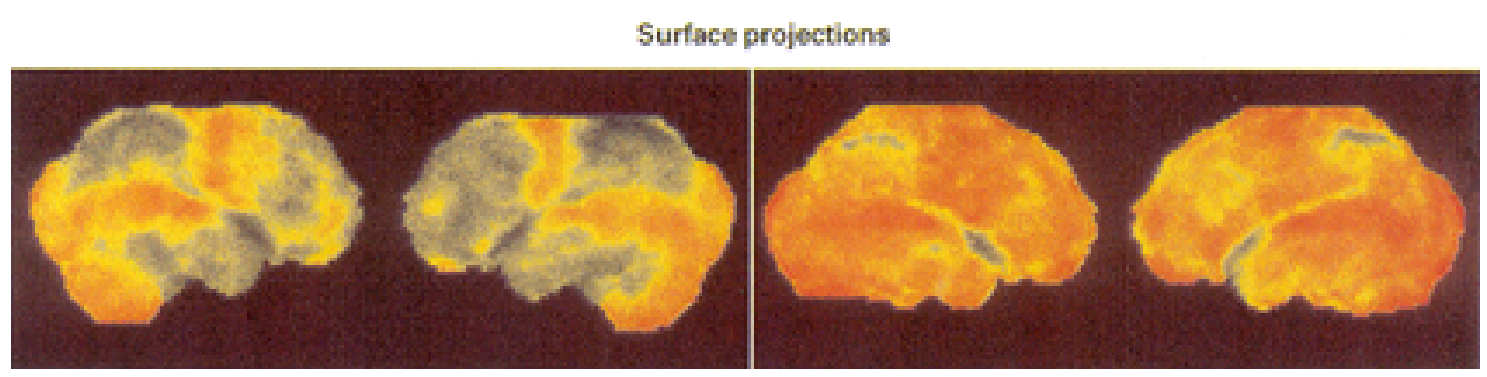

Fig. 1. FDG-PET 1 week after admission (study 1) and 5 weeks after starting the memantine treatment (study 2). The initial study showed a bilateral, symmetrical decrease in regional cerebral glucose consumption involving the frontal, parietal and temporal lobes. The second study, performed 5 weeks later, revealed a nearly normal pattern of glucose metabolism.

We started treatment with memantine for 5 weeks with a dosage up to a maximum of $30 \mathrm{mg} /$ day to improve memory and intellectual capacity. The patient was neuropsychologically tested before and retested after 5 weeks of treatment employing the same psychological tests. Improvement was seen in MMSE (Mini-Mental State Examination) [5] from 18 to 22 points, and CERAD Verbal Fluency (Consortium to Establish a Registry for Alzheimer's Disease) [6] from 10 to 12 points. CERAD Wordlist Recall improved from 0 to 5 and CERAD Drawing from 6 to 8 .

Parallel to psychological testing, an ${ }^{18} \mathrm{~F}$-fluorodeoxyglucose-positron emission tomography (FDG-PET) was performed 1 week after admission and 5 weeks after starting the memantine treatment, as extensively described elsewhere [7]. The initial study revealed a bilateral, symmetrical decrease in regional cerebral glucose consumption involving the frontal lobes and extended areas of the parietal and temporal lobes. The second study, performed 5 weeks later, revealed a nearly normal pattern of glucose metabolism (fig. 1). However, despite these findings and improvement of some intellectual and memory functions, severe memory deficits and disorientation persisted. The patient was recommended for continued inpatient care but no deterioration of the mental abilities was observed even 1 year after discharge.

This is the first report on memantine treatment in an ALD patient. To our knowledge, this is also the first report on FDG-PET in an ALD patient. Significant changes in cerebral blood flow after 5 weeks of memantine treatment were revealed using FDG-PET, which were paralleled by moderate improvements in neuropsychological testing. It supports the hypothesis that low-affinity NMDA antagonists have a neuroprotective effect even in manifest ALD patients $[3,4]$.

The PET findings prior to the memantine treatment were very similar to those in patients with AD. Both AD and ALD can be diagnosed after excluding other kinds of dementia and their FDG-PET findings may be similar. In our case, a 33-year history of severe alcohol intake was reported. The clinical course in AD is deteriorating over time whereas the mental abilities in this case remained stable under abstinent conditions and even improved slightly. This is very untypical for ALD. Furthermore, a well-known phenomenon is a spontaneous improvement in mental abilities in patients suffering from Korsakoff's syndrome or ALD [8]. This could not be proven in this case report. Thus, larger, controlled double-blind trials with this agent in ALD patients are needed to evaluate the use of memantine in alcohol-induced dementia syndromes.

\section{References}

1 Preuss UW, Soyka M: Wernicke-Korsakow syndrome: Clinical aspects, pathophysiology and therapeutic approaches. Fortschr Neurol Psychiatr 1997;65:413-420.

2 Tsai GC, Gastfried DR, Coyle JT: The glutamertergic basis of human alcoholism. Am J Psychiatry 1995;152:332-340.

3 Kornhuber J, Weller M: Psychotogenicity and N-methyl- $D$-aspartate receptor antagonism: Implications for neuroprotective pharmacotherapy. Biol Psychiatry 1997;41:135-144.

4 Muller WE, Mutschler E, Riederer P: Noncompetitive NMDA receptor antagonists with fast open-channel blocking kinetics and strong voltage dependency as potential therapeutic agents for Alzheimer's dementia. Pharmacopsychiatry 1995;28:113-124.

5 Folstein MF, Folstein SE, McHugh PR: 'Mini-Mental State'. A practical method for grading the cognitive state of patients for the clinician. J Psychiatr Res 1975;12:189-198.

6 Morris JC, Heyman A, Mohs RC, Hughes JP, van Belle G, Fillenbaum G, Mellits ED, Clark C: The Consortium to Establish a Registry for Alzheimer's Disease (CERAD). I. Clinial and neuropsychological assessment of Alzheimer's disease. Neurology 1989;39:1159-1165.

7 Bartenstein P, Minoshima S, Hirsch C, Buch K, Willoch F, Mosch D, Schad D, Schwaiger M, Kurz A: Quantitative assessment of cerebral blood flow in patients with Alzheimer's disease by SPECT. J Nucl Med 1997;38: 1095-1101.

8 Khudik VA: Dynamics of intellectual mnestic disorders in alcoholism (in Russian). Zh Nevropatol Psikhiatr Im S S Korsakova 1983;83:110-115.

U.W. Preuss, MD

Psychiatrische Klinik und Poliklinik

Ludwig-Maximilians-Universität München

Nussbaumstrasse 7, D-80336 München (Germany)

Tel. +49 895160 5740, Fax +49 $8951605748 / 4749$

E-Mail up@psy.med.uni-muenchen.de 